\title{
Left ventricular hypertrophy and arterial stiffness in essential hypertension
}

\author{
Yucel $\mathrm{C}^{1}$, Demir $\mathrm{S}^{2}$, Demir $\mathrm{M}^{3}$, Tufenk $\mathrm{M}^{4}$, Nas $\mathrm{K}^{5}$, Molnar $\mathrm{F}^{6}$, Illyes $\mathrm{M}^{7}$, Acarturk $\mathrm{E}^{1}$ \\ Cukurova University, School of Medicine, Department of Cardiology, Adana, Turkey. \\ demirkardiyoloji@hotmail.com
}

\begin{abstract}
AIM: The aim of this study was to evaluate the association between an increase in arterial stiffness and the development of left ventricular hypertrophy in essential hypertension patients.

MATERIALS AND METHODS: One hundred forty essential hypertension patients were included in the study. Patients were divided into two groups based on echocardiographic measurements; with left ventricular hypertrophy $(n=70)$ and without left ventricular hypertrophy $(n=70)$. The criterion for hypertrophy was accepted as an intraventricular septum and posterior wall thickness in diastole of $11 \mathrm{~mm}$ or above. Aortic stiffness values of the patients groups were measured noninvasively by arteriography through the brachial artery. Pulse wave velocity (PWV) measurements were used as indicators of arterial stiffness.

RESULTS: When compared to the group without left ventricular hypertrophy, elevated systolic blood pressure, mean blood pressure, and pulse pressure were located in the left ventricular hypertrophy group at a significant level $(p>$ 0.01). A statistically significant difference was not observed in the diastolic blood pressure and pulse measurements of the groups. Pulse wave velocity, the indicator of arterial stiffness, was elevated to a significant degree in the left ventricular hypertrophy group $(p>0.01)$. While a positive correlation was found between pulse wave velocity and left ventricle mass index, microalbuminuria, high sensitive C-reactive protein (Hs-CRP), and left ventricle enddiastolic volume, a negative correlation was found between pulse wave propagation velocity and left ventricle E/A. CONCLUSIONS: In conclusion, pulse wave analysis is a valuable method for predicting cardiac hypertrophy in essential hypertension (Tab. 6, Ref. 25). Text in PDF www.elis.sk. KEY WORDS: arterial stiffness, hypertension, left ventricular hypertrophy.
\end{abstract}

\section{Introduction}

Hypertension (HT) is a widespread and multifaceted public health issue throughout the world. Being the cause of other organ diseases, it has a significant impact on patient morbidity and mortality. Hypertension due to pressure load is a clinical indicator of progressive left ventricular hypertrophy (LVH) in hypertensive heart disease. Cardiovascular (CV) mortality risk increases approximately 7-9 fold in left ventricular hypertrophy patients (1). Arterial stiffness and pulsatile load are gradually being understood to make a significant contribution to the pathophysiology of HT and $\mathrm{CV}$ diseases (2). Structural changes in the vessel wall induced by the stress of high pressure due to hypertension and atherosclerosis cause aortic stiffness (3).

Particularly, the occurrence of arterial stiffness in large vessels was found to directly affect CV morbidity and mortality (4).

${ }^{1}$ Cukurova University, School of Medicine, Department of Cardiology, Adana, Turkey, ${ }^{2}$ Adana State Hospital, Karataş Yolu Adana Devlet Hastanesi, Yuregir/Adana, Turkey, ${ }^{3}$ Cukurova University Department of Cardiology, Saricam/Adana, Turkey, ${ }^{4}$ Cukurova University, Department of Cardiology, Adana, Turkey, ${ }^{5}$ Szent Janos Hospital, Radiology Department, Budapest, Hungary, ${ }^{6}$ Budapest University Technology and Echonomics, Budapest, Hungary, and ${ }^{7}$ Heart Institute, Pecs University Faculty of Medicine, Pecs, Hungary

Address for correspondence: S. Demir, MD, Adana State Hospital, Karatas Yolu Adana Devlet Hastanesi, 01270 Yuregir/Adana, Turkey.

Phone: +90.533.6271991
In studies examining aortic stiffness, predominantly pulse wave velocity (PWV) and augmentation index (Aix) were used to measure the "stiffness" index by invasive or non-invasive means (5). These parameters obtained non-invasively have been shown to be similar to those obtained invasively (6).

This study aimed to evaluate the association between left ventricular hypertrophy and arterial stiffness in hypertensive patients.

\section{Materials and methods}

Hypertensive patients $(\mathrm{n}=140)$ who applied to our clinic and were using calcium channel blocker (amlodipine) were included in the study. Patients were divided into two groups based on echocardiographic measurements; with left ventricular hypertrophy $(n=70)$ and without left ventricular hypertrophy $(n=70)$. We chose amlodipine because it may have a cardiovascular protective effect in addition to its strong blood pressure-lowering action and it has been reported to have several blood pressure-independent effects, including antioxidant activity, antiproliferative effects in vascular smooth muscle cells, and enhanced production of endothelial nitric oxide (7).

\section{Exclusion criteria}

Patients who had secondary causes of hypertension (renal failure, renal artery disease, pheochromocytoma, Conn syndrome 
etc.) concomitant atrial fibrillation, frequent ventricular extra ectopic beats, hypothyroidism, hyperthyroidism, respiratory diseases (COPD, chronic bronchitis, pulmonary embolism), primary pulmonary hypertension, acute coronary syndrome within the last 2 months, isolated right heart failure, congenital cardiac diseases and advanced valvular disease (mitral stenosis, aorta stenosis and failure), other chronic systemic disorders (diabetes, cirrhosis etc.v.b) were excluded from the trial. For all the patients included in the trial, data on the risk factors such as age, gender, hypertension, smoking, family history of cardiac diseases and the drugs received were recorded.

\section{Measurements of blood pressure and heart rate}

Blood pressure was measured with the assistance of a mercury sphygmomanometer. Pulse count was recorded by a oneminute measurement. Hypertension was defined as blood pressure $\geq 140 / 90 \mathrm{~mm} \mathrm{Hg}$.

\section{High sensitive CRP measerument}

Serum Hs-CRP was analyzed by immunonephelometric method with Beckman Coulter kit in Beckman Immage 800 (Beckman Coulter Inc., CA, USA) nephelometer. Analytical range of serum was HS-CRP $0.02-8.0 \mathrm{mg} / \mathrm{dL}(0.2-80.0 \mathrm{mg} / \mathrm{L})$ and analytical sensitivity for HS-CRP determination was $0.02 \mathrm{mg} / \mathrm{dL}(0.2 \mathrm{mg} / \mathrm{L})$.

\section{Microalbuminuria detection}

Microalbuminuria assessments were made at baseline A first morning voided spot urine sample for microalbumin (measured as the urinary albumin-creatinine ratio or UACR) was collected before administration of study treatment.

\section{Echocardiography}

All of the echocardiographic studies were performed using an Acuson Sequoia C-256 ultrasound machine. The overall, monodimensional left ventricular measurements and the bidimensional (apical 4- and 2-chamber) views were obtained according to the recommendations of the American Society of Echocardiography (8). All of the tracings were obtained and read by a single observer blinded to the clinical characteristics of the patients under observation. The presence of LVH was defined as left ventricular mass index $(\mathrm{LVMI})>49.2 \mathrm{~g} / \mathrm{m}^{2} .7$ in men and $>46.7 \mathrm{~g} / \mathrm{m}^{2} .7$ in women.

\section{Arterial stiffness measurement}

The Arteriograph cuff was applied on the left arm for measurement of blood pressure (BP), pulse wave propagation velocity (PWV) with the computerized portable device, Arteriograph (TensioMed Ltd.). It initially measures the BP in the upper arm oscillometrically and afterwards records a cuff pressure over the brachial artery that is $35 \mathrm{~mm} \mathrm{Hg}$ in excess of the systolic blood pressure (SBP) measured. The pressure fluctuations in the brachial artery are detected by the cuff. They are passed on to the computer and recorded and analyzed as pulse waves. The difference in time between the beginning of the first wave and the beginning of the second (reflected wave) is related to the measured distance from the jugulum to the symphysis, resulting in the $\mathrm{PWV}$ in $\mathrm{m} / \mathrm{s}$. The software of Arteriograph decomposes the early, late systolic and diastolic waves and also determines the onset and peaks of the waves. For PWV analysis, the onsets of the waves are determined by using first and second derivatives. To intensify the signal and thus attain better differentiation of the initial wave from the reflective wave, the Arteriograph only records and analyses the pulse waves when supra-SBP of $35 \mathrm{mmHg}$ has been attained.

\section{Statistical methods}

All analyses were performed with SPSS 16.1 statistical software package. Continuous variables in the group data are shown as mean \pm standard deviation (avg $\pm \mathrm{SD}$ ). Categorical variables are given as numbers and percentages. In the paired comparison of the groups, t-test was applied as parametric tests of independent samples. Mann-Whitney U and Kruskall-Wallis tests were applied as non-parametric tests. The Chi-square test was used in the comparison of categorical variables. Parametric or non-parametric (Pearson and Spearman) correlation analysis was performed in the investigation of associations among continuous variables. Statistical significance was accepted as $\mathrm{p}<0.05$.

\section{Results}

Observing patient demographic characteristics, a larger percentage of females $(67 \%)$ was found in the LVH (-) group (p $0.001) .36 \%$ of the $\mathrm{LVH}(+)$ group were females. A statistically significant difference did not exist between the groups with respect to age, smoking, BMI and HT time period (Tab. 1).

In the LVH (+) group urea, creatinine, hs-CRP, and microalbumin levels were found to be high; while serum sodium, throm-

Tab. 1. The comparison of group demographic characteristics.

\begin{tabular}{lccc}
\hline Characteristics of patients & $\mathrm{LVH}(-) \mathrm{n}=70$ & $\mathrm{LVH}(+) \mathrm{n}=70$ & $\mathrm{p}$ \\
\hline Age (years) & $55.9 \pm 11.55$ & $56.4 \pm 10.37$ & 0.845 \\
Smoker (n, \%) & $34(48.5)$ & $32(45.7)$ & 0.443 \\
Body mass index $\left(\mathrm{kg} / \mathrm{m}^{2}\right)$ & $30.17 \pm 4.89$ & $30.93 \pm 5.33$ & 0.762 \\
Hypertension duration (years) & $4.8 \pm 0.88$ & $5.01 \pm 0.92$ & 0.887 \\
\hline
\end{tabular}

Tab. 2. The comparison of group laboratory results.

\begin{tabular}{lccc}
\hline Laboratory results & LVH $(-) \mathrm{n}=70$ & $\mathrm{LVH}(+) \mathrm{n}=70$ & $\mathrm{p}$ \\
\hline Hemoglobin $(\mathrm{gr} / \mathrm{dL})$ & $13.2 \pm 1.2$ & $13.3 \pm 1.4$ & 0.121 \\
Glucose $(\mathrm{mg} / \mathrm{dL})$ & $92.3 \pm 16.1$ & $92.5 \pm 17.8$ & 0.812 \\
Creatinine $(\mathrm{mg} / \mathrm{dL})$ & $0.74 \pm 0.15$ & $0.97 \pm 0.16$ & 0.041 \\
Sodium $(\mathrm{mmol} / \mathrm{L})$ & $144.3 \pm 2.6$ & $141.9 \pm 3.6$ & 0.048 \\
hs-CRP $(\mathrm{mg} / \mathrm{dL})$ & $0.45 \pm 0.14$ & $2.1 \pm 0.53$ & 0.001 \\
e -GFR & $105.7 \pm 28.8$ & $86.8 \pm 20.5$ & 0.023 \\
Microalbuminuria $(\mathrm{mg} / \mathrm{l})$ & $115.7 \pm 58.9$ & $189.6 \pm 122.7$ & $<0.05$
\end{tabular}

hs-CRP - High-sensitivity C-reactive protein, e-GFR - Estimated glomerular filtration rate

Tab. 3. The comparison of group blood pressure and pulse values.

\begin{tabular}{lccc}
\hline Blood pressure and pulse results & $\mathrm{LVH}(-) \mathrm{n}=70$ & $\mathrm{LVH}(+) \mathrm{n}=70$ & $\mathrm{p}$ \\
\hline Systolic blood pressure (mmHg) & $148.5 \pm 13.6661$ & $145.4 \pm 16.0$ & 0.215 \\
Diastolic blood pressure (mmHg) & $85.5 \pm 12.1$ & $86.8 \pm 10.4$ & 0.433 \\
Mean BP (mmHg) & $103.2 \pm 11.4$ & $105.6 \pm 15.433$ & 0.036 \\
Pulse Pressure (mmHg) & $53.8 \pm 9.4$ & $58.3 \pm 10.5$ & 0.002 \\
Heart Rate (beats/ minute) & $75.4 \pm 10.2$ & $75.7 \pm 10.7$ & 0.305
\end{tabular}


Tab. 4. The comparison of group echocardiographic characeristics.

\begin{tabular}{lccc}
\hline Echocardiographic Results & LVH $(-) \mathrm{n}=70$ & LVH $(+) \mathrm{n}=70$ & $\mathrm{p}$ \\
\hline IVS diastolic thickness $(\mathrm{mm})$ & $9.5 \pm 0.5$ & $12.6 \pm 1.1$ & 0.001 \\
PW diastolic thickness $(\mathrm{mm})$ & $9.1 \pm 0.7$ & $11.7 \pm 0.7$ & 0.001 \\
LV end-diastolic dimension $(\mathrm{mm})$ & $45.0 \pm 3.2$ & $47.1 \pm 3.8$ & 0.006 \\
LV end-systolic dimension (mm) & $28.8 \pm 3.1$ & $31.0 \pm 3.3$ & 0.024 \\
LV mass index $\left(\mathrm{g} / \mathrm{m}^{2}\right)(\mathrm{LVMI})$ & $103.9 \pm 17.3$ & $148.6 \pm 23.9$ & 0.001 \\
LV ejection fraction (\%) & $64.5 \pm 2.8$ & $62.2 \pm 3.5$ & 0.017 \\
LA end-diastolic dimension (mm) & $34.6 \pm 2.2$ & $40.1 \pm 2.2$ & 0.001 \\
LA end-diastolic volume & $29.4 \pm 3.6$ & $53.7 \pm 12.4$ & 0.001 \\
Aortic end-systolic dimension (mm) & $30.9 \pm 2.2$ & $33.4 \pm 3.3$ & 0.001 \\
E (cm/s) & $73.7 \pm 14.4$ & $64.9 \pm 18.4$ & 0.002 \\
A (cm/s) & $62.8 \pm 15.1$ & $73.8 \pm 18.1$ & 0.001 \\
DT & $207.9 \pm 39.1$ & $258.9 \pm 69.1$ & 0.001 \\
IVRT & $106.4 \pm 14.1$ & $138.9 \pm 19.6$ & 0.001 \\
E/A & $1.17 \pm 0.2$ & $0.86 \pm 0.24$ & 0.001 \\
\hline IVRT - I
\end{tabular}

IVRT - Isovolumic relaxation time, LA - Left atrium, DT - Deceleration time, IVS - Interventricular septum, PW - Posterior wall, LV - Left ventricular

bocyte, and e-GFR values were low. Additional patient laboratory results are summarized in Table 2.

Significantly high systolic pressure, mean blood pressure, and pulse pressure were found in the left ventricular hypertrophy group. A statistically significant difference between the groups in diastolic blood pressure and pulse was not observed (Tab. 3).

In the echocardiographic examination interventricular septum, posterior wall, left ventricular dimensions, left ventricular mass index, and left atrium diameter and volume were larger to a significant degree in the LVH (+) group. Left ventricular ejection fraction was found to be low. Mitral flow PW (pulsed wave) was assessed by Doppler. Upon investigation of echocardiographic results, deceleration time, A, and IVRT of the LVH(+) group were greater to a significant degree; while E/A ratios and $\mathrm{E}$ were smaller (Tab. 4).

Arterial stiffness, indicated with PWV values, was higher at a statistically significant level in the group with left ventricular hypertrophy compared to the group without left ventricular hypertrophy (Tab. 5).

Positive correlations were found between PWV and left ventricular mass index, left atrium end-diastolic volume, hs-CRP, and microalbuminuria. A negative correlation was found between PWV and e-GFR, and the E/A ratio (Tab. 6).

\section{Discussion}

The primary finding of this study was the identification of an assocation between increased arterial stiffness, measured with PWV, and target organ damage. There is a growing understanding of the substantial contribution of arterial stiffness and pulsatile load on cardiovascular patients, HT, and heart failure pathophysiology (3). It was shown that increased conduit vessel stiffness results in elevated pulse wave velocity, and the wave's reflection in systole rather than diastole. While waves are reflected back in systole, forward waves are weakened and forward directed flow and pulse volume are reduced. The additional load placed on the fragile left ventricle triggers decompensation and arrhythmia (810). Furthermore, it has been demonstrated in animal models that the drift of retroreflective waves from diastole to systole causes ischemia in the presence or absence of epicardial stenosis (11).
Tab. 5. The comparison of the arterial stiffness marker PWV in the groups.

\begin{tabular}{lrrc}
\hline & $\mathrm{LVH}(-) \mathrm{n}=70$ & $\mathrm{LVH}(+) \mathrm{n}=70$ & $\mathrm{p}$ \\
\hline Pulse wave velocity $(\mathrm{m} / \mathrm{sn})$ & $10.4 \pm 1.2$ & $13.3 \pm 1.5$ & 0.001 \\
\hline
\end{tabular}

Tab. 6. The echocardiographic and laboratory parameters and assocations with PWV.

\begin{tabular}{lcc}
\hline PWV & $\mathrm{r}$ & $\mathrm{p}$ \\
\hline LV mass index $\left(\mathrm{g} / \mathrm{m}^{2}\right)(\mathrm{LVMI})$ & 0.745 & 0.001 \\
LA end-diastolic volume & 0.794 & 0.001 \\
LV E/A & -0.454 & $<0.05$ \\
Hs-CRP (mg/dL) & 0.813 & 0.001 \\
Microalbumin (mg/l) & 0.723 & $<0.05$ \\
\hline
\end{tabular}

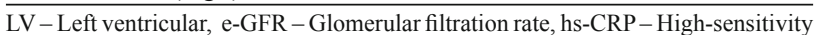
C-reactive protein, LA - Left atrium, PWV - Pulse wave velocity

Increased systolic blood pressure from arterial stiffness raises left ventricular afterload, which in turn raises myocardial workload and reduces diastolic blood pressure, thus impairing coronary perfusion and ultimately leading to subendocardial ischemia and fibrosis. A strong association has been demonstrated between increased arterial stiffness and target organ damage. Moreover, increased pulse wave velocity has been shown to be closely linked to CV mortality $(11,12)$. In systemic arterial hypertension, myocyte hypertrophy is induced by elevated afterload and activated neurohormonal mechanisms. Cardiac fibrosis ensues in intramyocardial coronary arteries as a result of medial hypertrophy and collagen deposition. These modifications lead to left ventricular hypertrophy. Left ventricular hypertrophy is an independent predictor of morbidity and mortality in hypertensive patients. It predisposes patients to congestive heart failure, ischemic stroke, atrial fibrillation, ventricular tachycardia, and embolic stroke $(13,14)$. A study by Giovanna Leoncini et al. found a significant positive assocation between elevated arterial stiffness and left ventricular mass index (13). An assocation was identified by Nitta et al between left ventricular hypertrophy, elevated arterial stiffness, and vascular calcification in hemodialysis patients (15). A statistically significant increase in PWV and Aix was confirmed in the left ventricular hypertrophy hypertensive group compared to the group without left ventricular hypertrophy. In a Framingham study a significant association was identified between left ventricular hypertrophy and systolic hypertension (16). In our study, systolic and mean blood pressure, as well as pulse pressure were found to be higher at a significant level in the group with developing LVH and elevated left ventricular mass index. However, a difference between the groups in diastolic blood pressure was not observed. Left venricular systolic and diastolic malfunction in heart failure are important factors in lifespan. Diastolic function is thought to be more greatly impaired in patients with left ventricular hypertrophy (17). Compared to the hypertension group without hypertrophy, the diastolic dysfunction indicator in the left ventricular hypertrophy group was associated with multiple echocardiographic parameters at a statistically significant level. In the LVH (+) group PWV, left ventricular mass index, and left atrium end-diastolic volume were positively associated. On the other hand, PWV was negatively associated with the $\mathrm{E} / \mathrm{A}$ ratio. 
Being associated with many metabolic and non-metabolic risk factors, microalbuminuria is a common microvascular damage indicator independent of other risk factors in patients with essential HT, and is estimated as the primary determinant of CV mortality. Pontremoli et al. reported in a study of 787 patients with essential HT that the association between the incidence of microalbuminuria and the development of CV disease could indicate urinary albumin excretion as a reliable marker in the diagnosis of CV (16). In a study of 205 essential HT patients, Tsai et al. found that arterial stiffness was greater to a statistically significant degree in the group with LVH and proteinuria (19). Consequently, measurement of urinary albumin excretion is critical for hypertensive patients (20). In the Cerasola et al. study, it was found that patients with microalbuminuria had a higher blood presure and peripheral PWV in 24 hours. In our study microalbuminuria was high in the $\mathrm{LVH}(+)$ group, and was postively correlated with PWV.

Regardless of the presence of other risk factors, high hs-CRP values are independently accompanied by increased risk of $\mathrm{CV}$ event (21-22). Kampus et al found that with Aix increase, hs-CRP, and leukocyte count were positively associated (23). Rotterdam identified a strong association between hs-CRP and carotid-femoral PWV (24). When Kim et al. separated patients into three groups according to hs-CRP values a positive correlation between hs-CRP and PWV was determined (26). Hs-CRP's link to vessel stiffness was independent of age, SBP, sex, pulse, glucose values, lipid parameters, and HT patient medication use. Consequently, hs-CRP could be considered a practical marker in the assessment of arterial stiffness and in the follow-up treatment of hypertensive patients (25). In our study as well, the hs-CRP level was high in patients with $\mathrm{LVH}$, and a significant correlation between PWV and hs-CRP level was identified.

\section{Conclusion}

In our study, target organ damage was determined in left ventricular hypertrophy patients with elevated arterial stiffness. In conclusion, pulse wave analysis is a valuable method in predicting cardiac hypertrophy in essential hypertension. Arterial stiffness should be taken into account when planning therapeutic strategies for prevention of left ventricular hypertrophy in hypertensive patients.

\section{Limitations}

One hundred forty patients were included in our study. Therefore, studies with larger sample sizes are needed. This is a crosssectional study. Longitunal studies are need to determine the relation between PWV and left ventricular hypertrophy.

\section{References}

1. Kawaguchi M, Hay I, Fetics B. Combined ventricular systolic and arterial stiffening in patients with heart failure and preserved ejection fraction: implications for systolic and diastolic reserve limitations. Circulation 2003; 107: 714-720.
2. Kawaguchi M, Hay I, Fetics B, Kass DA. Combined ventricular systolic and arterial stiffening in patients with heart failure and preserved ejection fraction: implications for systolic and diastolic reserve limitations. Circulation 2003; 107: 714-720.

3. O’Rourke MF, Staessen JA, Vlachopoulos C, Duprez D, Plante GE. Clinical applications of arterial stiffness; definitions and reference. Intern J Epidemiol 2004; 33: 161-162.

4. Devereux RB, Alderman MH. Role of preclinical cardiovascular disease in the evolution from risk factor exposure to development of morbid events. Circulation 1993; 88: 1444-1455.

5. Iketani T, Iketani Y, Takazawa K, Yamashina A. The influence of the peripheral reflection wave on left ventricular hipertrophy in patients with essential hypertension. Hypertens Res 2000; 23: 451-458.

6. Tsai WC, Lin CC, Huang YY, Chen JY, Chen JH. Association of increased arterial stiffness and inflammation with proteinuria and left ventricular hypertrophy in non-diabetic hypertensive patients. Blood Pressure 2007; 16: 270-275.

7. Ding Y, Vaziri ND. Nifedipine and diltiazem but not verapamil upregulate endothelial nitricoxide synthase expression.] Pharmacol Exp Ther 2000; 292: 606-609.

8. Cheitlin MD, Armstrong WF, Aurigemma GP, Beller GA, Bierman FZ, Davis JL, Douglas PS, Faxon DP, Gillam LD, Kimball TR, Kussmaul WG, Pearlman AS, Philbrick JT, Rakowski H, Thys DM, Antman EM, Smith SC Jr, Alpert JS, Gregoratos G, Anderson JL, Hiratzka LF, Faxon DP, Hunt SA, Fuster V, Jacobs AK, Gibbons RJ, Russell RO; ACC; AHA; ASE. ACC/AHA/ASE 2003 Guideline Update for the Clinical Application of Echocardiography: summary article. A report of the American College of Cardiology/American Heart Association Task Force on Practice Guidelines (ACC/AHA/ASE Committee to Update the 1997 Guidelines for the Clinical Application of Echocardiography). J Am Soc Echocardiog 2003; 16 (10): 1091-1110.

9. Westerhof N, O’Rourke MF. Haemodynamic basis for the development of left ventricular failure in systolic hypertension and for its logical therapy. J Hypertens 1995; 13: 943-952.

10. Arnold JM, Marchiori GE, Imrie JR, Burton GL, Pflugfelder PW, Kostuk WJ. Large artery function in patients with chronic heart failure. Studies of brachial artery diameter and hemodynamics. Circulation1991; 84: 2418-2425.

11. Meaume S, Benetos A, Henry OF, Rudnichi A, Safar ME. Aortic pulse wave velocity predicts cardiovascular mortality in subjects 70 years of age. Arterioscler Thromb Vasc Biol 2001: 21: 2046-2050.

12. Mitchell GF, Parise H, Benjamin EJ, Larson MG, Keyes MJ, Vita JA, Vasan RS, Levy D. Changes in arterial stiffness and wave reflection with advancing age in healthy men and women: the Framingham Heart Study. Hypertension 2004; 43: 1239-1245.

13. Giovanna L, Elena R, Francesca V, Valentina V, Angelica P, Valeria F, Novella C, Cinzia T, Giacom D, Roberto P. Increased Ambulatory Arterial Stiffness Index Is Associated With Target Organ Damage in Primary Hypertension. Hypertension.2006; 48: 397-403.

14. Aronson D, Burger AJ. Relation between pulse pressure and survival in patients with decompensated heart failure. Am J Cardiol 2004; 15: 93: 785-788.

15. Nitta K, Akiba T, Uchida K, Otsubo S, Otsubo Y, Takei T, Ogawa T, Yumura W, Kabaya T, Nihei H. Left ventricular hypertrophy is associated with arterial stiffness and vascular calcification in hemodialysis patients. Hypertens Res 2004; 27: 47-52. 


\section{4-718}

16. Sagie A, Benjamin EJ, Galderisi M, Larson MG, Evans JC, Fuller DL, Lehman B, Levy D. Echocardiographic assessment of left ventricular structure and diastolic filling in elderly subjects with borderline isolated systolic hypertension (the Framingham Heart Study). Amer J Cardiol 1993; 72 (9): 662-665.

17. Zabalgoitia M, Rahman SNU, Haley WE. Disparity between diastolic mitral flow characteristics and left ventricular mass in essential hypertension. Am J Cardiol 1997; 79: 1255-1258.

18. Pontremoli R, Nicolella C, Viazzi F, Ravera M. Microalbuminuria is an early marker of target organ damage in essential hypertension. Am J Hypertens 1998; 11: 430-438.

19. Tsai WC, Lin CC, Huang YY, Chen JY, Chen JH. Association of increased arterial stiffness and inflammation with proteinuria and left ventricular hypertrophy in non-diabetic hypertensive patients. Blood Pressure 2007; 16: 270-275.

20. Seco ML, Rus A, Sierra M, Caballero M, Borque L. Determination of serum cystatin $\mathrm{C}$ in patients with essential hypertension. Nephron 1999; 81 (4): 446-447.
21. Cerasola G, Cottone S, Mule G. Microalbuminuria, renal dysfunction and cardiovascular complication in essential hypertension. J Hypertens 1996; 14: 915-920.

22. Ross R. Atherosclerosis - antinflammatory disease. N Engl J Med 1999; 340: 115-126.

23. Kampus P, Muda P, Kals J. The relationship between inflammation and arterial stiffness in patients with essential hypertension. Intern J Cardiol 2006; 46-51.

24. Mattace-Raso FU, van der Cammen TJ, van der Meer IM. CRP and arterial stiffness in older adults: the Rotterdam Study. Atherosclerosis 2004; 176: 111-116.

25. Jung-Sun K, Tae Soo K, Jin- BK. Significant association of C-reactive protein with arterial stiffness in treated non-diabetic hypertensive patients. Atherosclerosis 2007; 192 (2): 401-406.

Received February 2, 2015. Accepted February 15, 2015. 